\title{
Development of an experimental apparatus and a data analysis protocol for the test of hydrogen volume and flow meters in controlled environmental conditions
}

\author{
Francesco Battistella ${ }^{1}$, Antonino Ravidà ${ }^{1}$, Massimo Mastromatteo ${ }^{2}$, Roberto Simone Pinna ${ }^{2}$, \\ and Gianluca Valenti ${ }^{1, *}$ \\ ${ }^{1}$ Politecnico di Milano, Dipartimento di Energia, Via Lambruschini, 4a, 20156 Milano (MI), Italy \\ ${ }^{2}$ Pietro Fiorentini S.p.A., Via Fermi 8/10, 36057 Arcugnano (VI), Italy
}

\begin{abstract}
The decarbonization of the residential sector is fundamental for energy transition. In this context, it is promising the introduction of hydrogen in natural gas networks in specific hydrogen districts. Accordingly, hydrogen meters are needed for accounting the fuel consumptions. The topic of this work is the development and construction of an experimental apparatus for testing safely hydrogen volume and flow meters up to $24 \mathrm{~m}^{3} / \mathrm{h}$ (referred to standard conditions) in controlled environmental conditions, between -25 and $+55{ }^{\circ} \mathrm{C}$ (and beyond). The apparatus realized can test up to four volume and flow meters in a climatic chamber while processing air or pure hydrogen or methane. Methanehydrogen mixtures can be tested connecting simply bottles with synthetic blends. The aim is to verify the measurement accuracy of the meters under test. A dedicated data analysis protocol featuring statistical process control is developed to monitor the stability of the system during the test. It exploits statistical indicators representing the autocorrelation, the normality of residuals of the mean value and the lag plot. The apparatus is realized, and it complies with the leakage limits set by indications in literature. A new ultrasonic domestic meter is tested in the apparatus. It has been developed by Pietro Fiorentini S.p.A. in the framework of the Hy4Heat project. Its error trends measured at all temperatures comply with the limit of $3.5 \%$ between 0.12 and $2 \mathrm{~m}^{3} / \mathrm{h}$ and $2 \%$ between 2 and $20 \mathrm{~m}^{3} / \mathrm{h}$, as imposed by legislations.
\end{abstract}

\section{Introduction}

The process of energy transition involves the decarbonization of hard-to-abate sectors. Among these, households are responsible of roughly the $20 \%$ of the greenhouse gas emissions [1]. This fact promotes the interest in hydrogen technologies as they can enable the decarbonization of this sector. In this context, the injection of hydrogen in natural gas networks appears the promising solution. Hydrogen could replace natural gases or could be blended with them to facilitate the transition between fuels [2]. Consequently, hydrogen can

\footnotetext{
*Corresponding author: gianluca.valenti@polimi.it
} 
be introduced in households with minimal disruption for the end-users. This practice is already investigated, and it is expected to be implemented in hydrogen districts.

A hydrogen district is a region where the natural gas network is converted for delivering hydrogen to end-users. The feasibility of such districts is being investigated mostly in Europe and, particularly, in the United Kingdom (UK). Examples of the projects are the H21 suite in the UK or municipalities in the Netherlands as part of the Natural Gas Free District Program $[3,4]$. In Italy, hydrogen valleys are presented in the recent recovery plan named "Piano Nazionale di Ripresa e Resilienza" for the decarbonization of critical sectors [5]. Such districts will increase the demand for equipment to manage a hydrogen network. Accordingly, hydrogen volume and flow meters are needed to account the fuel consumptions.

The tests on volume and flow meters are ruled by legislations. The main reference for the regulation of the tests is the European Measuring Instruments Directive (MID), ruling general metrology requirements for measuring instruments [6]. On such legislation is based the European Standard EN 14236 [7]. It states all the tests and safety standards for the ultrasonic flow meters. Indications from international organisations are also considered when designing and realizing a test bench. The German Department named "Physikalisch-Tecnische Bundesanstalt" gives suggestions for setting the apparatus and about its performances [8]. Further indications on the testing of residential meters with internal temperature compensation come from the Organisation Internationale de Métrologie Légale [9].

The topic of this work is the development and construction of an experimental apparatus for testing hydrogen volume and flow meters. It must be able to perform tests in standard conditions as well as in extreme hot or cold environmental conditions. The volume and flow meters can be operated in a range of temperatures from $-25{ }^{\circ} \mathrm{C}$ to $+55^{\circ} \mathrm{C}$. The apparatus can process air and methane flow rates in a range from 0.04 to $7.2 \mathrm{~m}^{3} / \mathrm{h}$ as well as hydrogen from 0.12 to $24 \mathrm{~m}^{3} / \mathrm{h}$, where volumes are referred to standard conditions of $15^{\circ} \mathrm{C}$ and $101.325 \mathrm{kPa}$. Together with the apparatus, it is developed an operational procedure.

The present work also features the development of a data analysis protocol with statistical process control. The subject is deeply investigated by Montgomery in a dedicated textbook [10]. It introduces the important statistical parameters of a sample and the approaches to deal with autocorrelated data. Information on such parameters is also available on the website of the National Institute of Standards and Technology [11]. This website describes the main statistical indicators checked to assess the stability of samples. The data analysis protocol implemented increases the reproducibility of the results verifying the conditions during each test. This way, misleading information deriving from instabilities in the system is avoided.

The aim of the system is to verify the measurement accuracy of hydrogen volume and flow meters with respect to a reference system. This assessment must be performed even under extreme environmental conditions, as temperature variation. The operating conditions of the tested meters are recorded with a reference system. Finally, the readings of the meters under test are compared with the ones of reference thermal mass flow meters.

The apparatus is placed in the Laboratory of Energy Conversion and Storage of Politecnico di Milano. It can process air from a network and hydrogen or methane from bottles. The meters can be tested in standard or extreme environmental conditions at an operating relative pressure of roughly $2 \mathrm{kPa}$, in a dedicated climatic chamber. The data analysis protocol computes some statistical indicators to assess the stability of each sample. Additionally, it converts the readings in comparable unit of measurements, and it computes the deviations between the tested meters and the reference thermal mass flow meters. Finally, a case study is presented to mark the functionality of the whole system.

This paper features a description of the experimental apparatus and the safety systems in the next section. It also presents the testing procedure and the instrumentation exploited, the data analysis protocol, the results from a case study where volume and mass flow meters have been calibrated with the apparatus. The conclusions of the work are drawn in the final section. 


\section{Experimental apparatus}

This section describes the experimental apparatus introduced above and the testing procedure to operate it. It also reports all the instrumentation exploited as reference as well as the safety systems included in the apparatus.

\subsection{Description of the apparatus}

The apparatus can test up to four volume and flow meters in controlled environmental conditions as part of the apparatus itself is located inside a climatic chamber. The tests can be executed with air for an easier comparison with diverse testing facilities or with methane or with pure hydrogen. Methane-hydrogen mixtures can be introduced connecting simply bottles with synthetic blends of interest to the methane line. Such mixtures might be realized with additional dedicated mass flow controllers on the methane and hydrogen lines. Furthermore, the apparatus can flow nitrogen to flush residuals of flammable gases. Safety is ensured by leakage sensors, a diluting system, and a procedure for the operation. Figure 1 shows the process flow diagram of the apparatus, divided in sections with different tasks:

- Section 1 connects to the delivery lines and regulates the pressure,

- Section 2 hosts inside a climatic chamber the tested volume and flow meters

- Section 3 controls the flow rate and vents the processed gases in atmosphere.

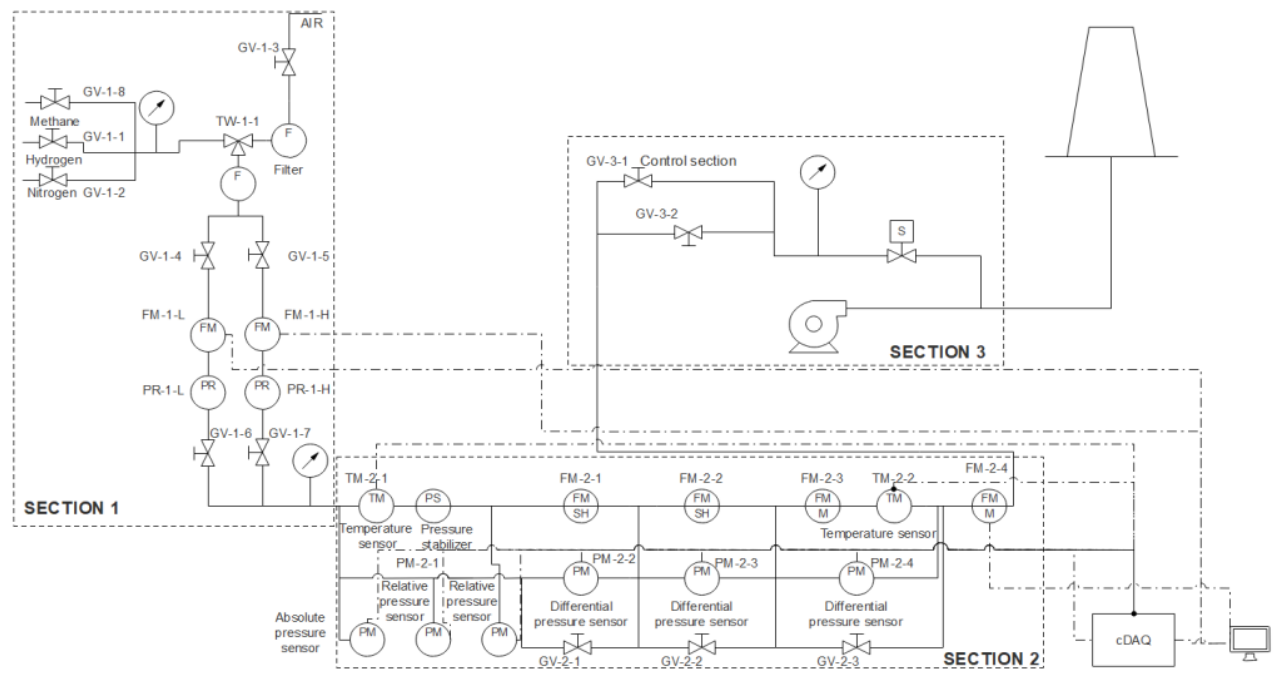

Fig. 1. Process flow diagram of the apparatus

Section 1 performs the reference measurement of the flow rate and the pressure regulation of the fluid. The connections to the lines of air and technical gases have a relative pressure of $500 \mathrm{kPa}$. The technical gases exploited are pure hydrogen, methane, and nitrogen for purging. A three-way valve enables the feeding with technical gases or compressed air. Moreover, the user can select the technical gas to process with ball valves. Section 1 also features two parallel branches for processing either high or low flow rates. Each branch contains a reference thermal mass flow meter, a pressure regulator and ball valves to isolate the equipment for maintenance. The pressure regulators are manufactured by Pietro Fiorentini S.p.A., model FE, and they are commonly used for natural gas residential distribution networks. All the leakages in the section are monitored with a pressure gauge. Finally, the gases are delivered to section 2 of the apparatus with a relative pressure of roughly $7 \mathrm{kPa}$. The overview of section 1 is reported in figure 2 . 


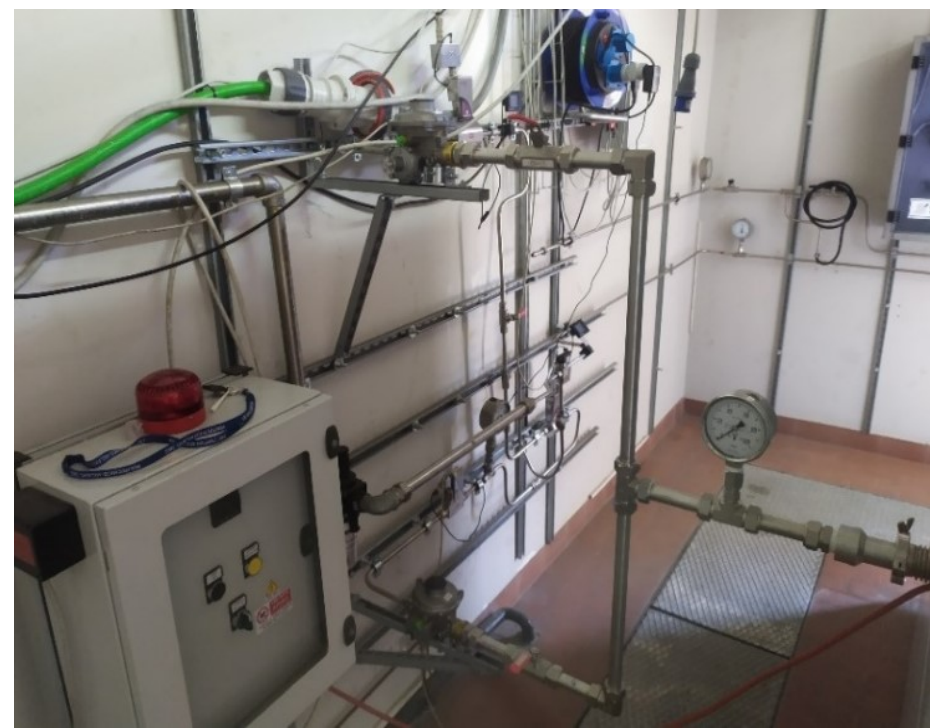

Fig. 2. Section 1 of the apparatus with the lines of air and technical gases as well as reference thermal mass flow meters and pressure regulators.

Section 2 contains the volume and flow meters under test, stabilizes their operating pressure and monitors the operating conditions. The volume and flow meters are operated at a relative pressure of $2 \mathrm{kPa}$ selected with a pressure stabilizer. This section works at controlled environmental temperature in a range from $-25^{\circ} \mathrm{C}$ to $+55^{\circ} \mathrm{C}$. A climatic chamber allows the selection of the environmental temperature for each test. The positioning of the pipelines inside the climatic chamber is optimized for achieving the best thermalization of the test gas. All the operating conditions are monitored with reference sensors. They measure the stream temperature, the absolute operating pressure, the relative pressures upstream and downstream the pressure stabilizer and the differential pressures upstream and downstream each tested meter. Ultimately, inside the climatic chamber is placed a leakage detector. The overview of section 2 is reported in figure 3 .

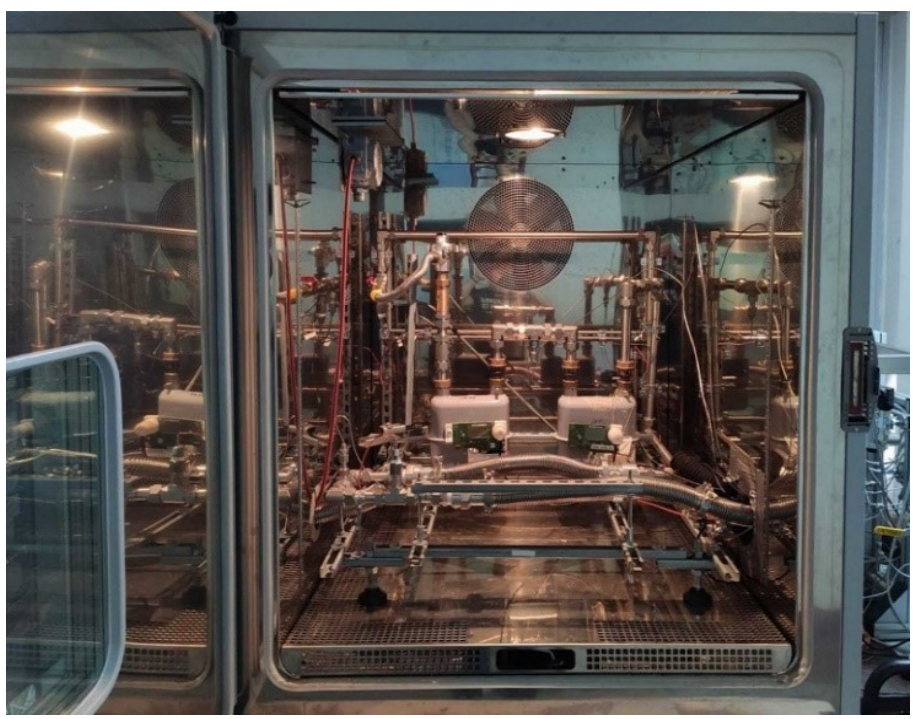

Fig. 3. Section 2 of the apparatus and the climatic chamber to control the testing conditions. 
Section 3 features the control of the flow rate and dilutes the stream for the safe venting of flammable gases in the atmosphere. The control of the flow rate occurs with two parallel valves. A ball valve for coarse regulation and a needle valve for fine regulation. Afterwards, the stream is diluted in a dedicated system. It is developed by SAPIO and it dilutes hydrogen or methane in a monitored stream of air with a specific nozzle. Consequently, it is guaranteed the generation of a uniform mixture below the flammability limit. The diluting system features an independent control system for providing the maximum operational safety. Finally, the generated non-flammable mixture is vented in the stack. The overview of section 3 and of the data acquisition system is reported in figure 4 .

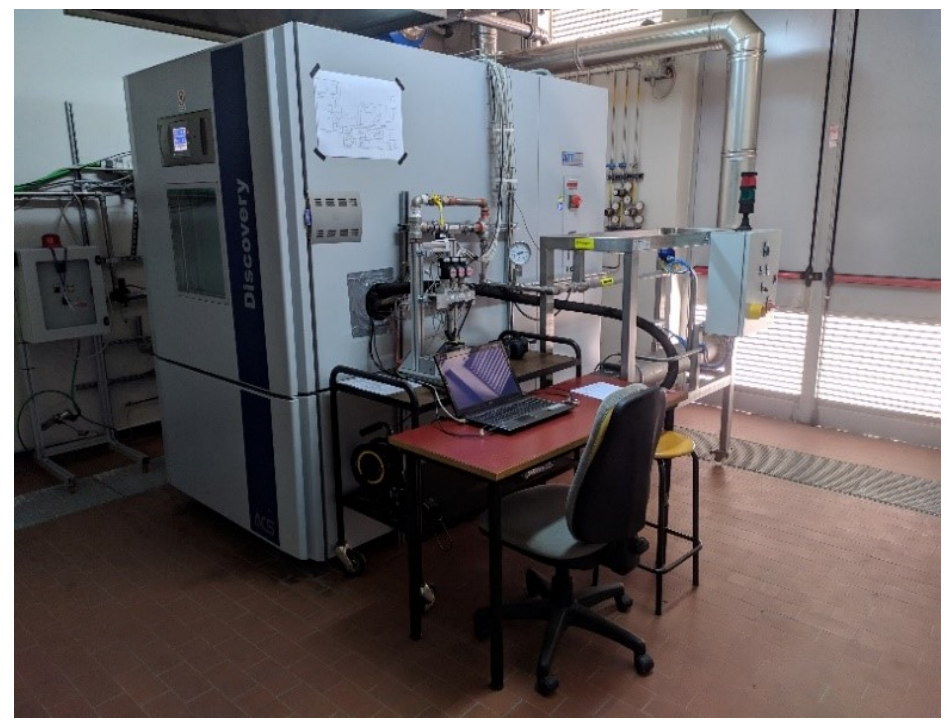

Fig. 4. Section 3 of the apparatus and the data acquisition system

The climatic chamber adopted for the apparatus is an Angelantoni Discovery DY 1200. It can produce temperatures from $-40{ }^{\circ} \mathrm{C}$ to $+180{ }^{\circ} \mathrm{C}$ and relative humidities from $10 \%$ to $98 \%$. Moreover, it might operate in steady state or following user-defined cycles. The whole data acquisition is performed with a dedicated laptop. The volume and flow meters under test are directly connected and the interact thanks to a dedicated Graphical User Interface. The reference thermal mass flow meters and the reference sensors are connected to a National Instruments cDAQ. All their data are collected with an internally developed National Instruments LabVIEW code. The information from the reference meters and sensors are transmitted as voltage signals for the temperature sensors and as current signals for the other instruments. The characteristics of the reference instruments and sensors are listed in table 1.

The safety systems in the testing cell and the apparatus spot leakages of flammable gases. They warn the user before having the formation of hazardous mixtures. The testing cell includes a high-level safety system with diverse leakage sensors placed in target areas. When a leakage is detected, all the delivery lines of technical gases are automatically closed with solenoid valves. The apparatus also features an own leakage sensor, placed inside the climatic chamber. It is a Dräger PEX 3000 and can detect flammable gases below the explosive limit $(0-100 \%$ of Lower Explosive Limit). The maximum safety of operation is achieved also following the developed testing procedure, presented in the next section. 
Table 1. Instrumentation installed on the apparatus for monitoring the operational conditions and measure the processed flow rate. Volumes are referred to normal unit $\left(0{ }^{\circ} \mathrm{C}, 101.325 \mathrm{kPa}\right)$. F.S. stands for full scale, while R.V. for read value.

\begin{tabular}{cccc} 
Parameter & Instrument & Range & Uncertainty \\
Low flow rates & $\begin{array}{c}\text { Bronkhorst } \\
\text { F112-AI }\end{array}$ & $\begin{array}{c}0-9 \mathrm{~m}^{3} / \mathrm{h}-\text { air. } \\
0-6.4 \mathrm{~m}^{3} / \mathrm{h}-\text { methane. } \\
0-8.4 \mathrm{~m}^{3} / \mathrm{h}-\text { hydrogen }\end{array}$ & $\begin{array}{c}0.1 \% \mathrm{~F} . \mathrm{S} . \\
0.5 \% \mathrm{R} . \mathrm{V} .\end{array}$ \\
\hline High flow rates & $\begin{array}{c}\text { Bronkhorst } \\
\text { F113-AI }\end{array}$ & $\begin{array}{c}0-10.5 \mathrm{~m}^{3} / \mathrm{h}-\text { air, methane } \\
0-30 \mathrm{~m}^{3} / \mathrm{h}-\text { hydrogen }\end{array}$ & $\begin{array}{c}0.1 \% \mathrm{~F} . \mathrm{S} . \\
0.5 \% \mathrm{R} . \mathrm{V} .\end{array}$ \\
\hline $\begin{array}{c}\text { Stream temperature } \\
\text { Pt 100 sensor }\end{array}$ & $-200-850^{\circ} \mathrm{C}$ & $0.3{ }^{\circ} \mathrm{C}$ \\
\hline $\begin{array}{c}\text { Differential } \\
\text { pressure }\end{array}$ & $\begin{array}{c}\text { BD|SENSORS } \\
\text { DS 233 }\end{array}$ & $0-6 \mathrm{kPa}$ & $1 \% \mathrm{~F} . \mathrm{S}$. \\
\hline Absolute pressure & $\begin{array}{c}\text { Drück } \\
\text { UNIK 5000 }\end{array}$ & $80-120 \mathrm{kPa}$ & $0.04 \% \mathrm{~F} . \mathrm{S}$. \\
\hline
\end{tabular}

\subsection{Test procedure}

The main motivation for developing the testing procedure is to establish a best practice to increase the safety and the reproducibility of laboratory operations. The aim is also to avoid the formation of hazardous mixtures in the apparatus and to prevent an uncontrolled leakage of flammable gases. Moreover, the test procedure delivers a higher reliability of the results and a more efficient operation of the apparatus. Together with the test procedure, the user must follow a test plan agreed before each test session.

Eventual leakages from the apparatus are detected with a leakage test. It also guarantees the maximum accuracy of the data gathered. Indeed, leakages between the reference thermal mass flow meter and the meters under test lead to inaccurate information in the readings. The leakage test is performed checking the pressure in each section of the apparatus with closed upstream and downstream valves. This test is included in the procedure and it is repeated before each test session. It must replicate the results obtained in the validating test after the construction of the apparatus. In such test, the leakage has been equal to $0.0 \pm 1.1 \mathrm{~Pa} / \mathrm{min}$ with the apparatus filled with hydrogen. The indications in literature require a leakage lower than $6.67 \mathrm{~Pa} / \mathrm{min}$ [8]. Each leakage test is first carried out with compressed air. This enables a first check on the integrity of the fittings. Subsequently, the apparatus is flushed with nitrogen. It is a standard practice for the avoidance of the formation of hazardous mixtures. Afterwards, the leakage test is performed with hydrogen. If any leakage is still present, it is identified with a Testo 316-2 portable hydrogen detector and it is fixed improving the connections of the apparatus. The procedure for the leakage test with hydrogen is repeated until it gives the results obtained in the validation test.

Each test procedure starts with the thermalization of the apparatus. The climatic chamber is set at the desired temperature and the thermal transient happens in some hours. All the equipment must be at thermal equilibrium before starting the tests. Moreover, absence of leakages must be checked before processing flammable gases. The test is performed as presented in the previous paragraph. Afterwards, the apparatus is filled with hydrogen. For processing compressed air in a test session, it is mandatory to flush the apparatus with nitrogen for several minutes to avoid contact with hydrogen. The first test of the plan is with zero flow rate. It enables the understanding of the undisturbed behaviour of the meters and the final check on the absence of leakages. Ultimately, the flow rate for each test can be selected with the valves in section 3 following the test plan. When the flow rate is stable, the 
data acquisition system can be launched. The duration and the flow rate to be processed during each test are defined in the test plan.

The test plan is defined as a function of the characteristics of the volume and flow meters under test. The aim is avoiding waste of technical gases or damages to the meters. The flow rates included in the test plan are defined by the legislation [7]. They are expressed as a function of the minimum and maximum flow rates. Such values must be agreed with the producer of the volume and flow meter. The duration of each test is defined to obtain enough data in steady state for the application of the statistical process control.

\section{Data analysis protocol}

This section presents the statistical process control and the charts of the indicators exploited for its execution. They represent the autocorrelation, the normality of residuals and the lag plot of a sample. In the section are also presented the autoregressive model for autocorrelated samples and the Shewhart control charts. Finally, the output of the data analysis process is introduced. It involves the mean deviations of the readings of the meters.

\subsection{Statistical process control}

The statistical process control is performed controlling peculiar charts and statistical indicators of the steadiness of each sample as presented in literature [10,11]. The first chart considered is the Shewhart control chart. It allows to detect shifts or trends or outliers in a sample. This chart is applied to samples with low autocorrelation or to the residuals of the autoregressive model. It can only be applied if a sample follows a normal distribution [12]. The Shewhart control chart includes the control limits computed as a function of the sample standard deviation. It is constructed plotting the data in a sample and connecting them. Finally, the control limits are stacked to highlight points out of control.

After the Shewhart control chart, two charts to monitor the stability of the sample are plotted even though they do not represent statistical indicators. Firstly, the moving average plot is useful to have a first check on the stability of the information gathered. Each point of the plot is obtained computing the average value of the 10 points that precede the corresponding in the sample. The moving average value is plotted against the index of the corresponding point. Secondly, the scatter plot represents the value of each point in the sample against its index. It helps in spotting eventual outliers generated by faults.

The first statistical indicator considered is the autocorrelation. It is suggested to detect non-random behaviours of a sample [11]. It represents the correlation of different data as a function of the lag in between them. The lag is the number of sampling periods dividing two data points in the sample. When a sample is random, the autocorrelation is close to zero for all lags. Oscillations in the autocorrelation indicate a possible instability of the sample. Therefore, further investigations must be performed.

Another indicator investigated is the normality of the residuals of the mean value. When such residuals are normally distributed, the sample can be described with its mean value. The detection of any structure in the residuals implies that the mean does not account for such structure. The normality of residuals of the mean is investigated with two charts. A first chart is the histogram of the residuals. They are divided in classes and the height of each bar is proportional to the occurrence of a class of residuals. The second chart analysed is the normal probability plot. It compares the residuals of the mean with values from a normal distribution. The latter form a straight line and points of a normally distributed sample lie on such line.

Further investigation on the presence of a structure in the sample is performed with the lag plot. Each point of the lag plot is constructed with the horizontal coordinate equal to the 
value of a point in the sample and the vertical coordinate equal to the value of the preceding point in the sample. A random sample does not show any structure in the lag plot.

Finally, if the autocorrelative structure is not negligible, it shall be accounted with an Auto Regressive Integrated Moving Average model. The data analysis protocol applies a first order autoregressive model to autocorrelated samples. It features the regression of the sample with respect to itself one lag before. With this approach, the autocorrelative structure is removed from the sample. Therefore, the residuals between the sample and the model are investigated with a conventional control chart for spotting any irregularities [10].

\subsection{Output of the data analysis process}

After the punctual analysis of the statistical process control, the data analysis protocol considers the mean values of samples. All the readings of the reference thermal mass flow meters and of the volume and mass flow meters are converted in standard units. The unit is referred to conditions with $15^{\circ} \mathrm{C}$ and $101.325 \mathrm{kPa}$. The converted readings are exploited to compute the mean flow rate value of each sample. Such values are compared with the readings of reference thermal mass flow meters. Moreover, it is computed the relative deviation between the meter under test and the reference meters, $E_{\%}$ (dimensionless), is:

$$
E_{\%}=100 * \frac{\bar{Q}_{m u t}-\bar{Q}_{r e f}}{\bar{Q}_{r e f}}
$$

where $\bar{Q}$ refers to the mean flow rate of the meter under test (mut) and the reference thermal mass flow meter (ref), both expressed in standard units $\left(+15^{\circ} \mathrm{C}\right.$ and $\left.101.325 \mathrm{kPa}\right)$.

The deviation values are exploited in the first stage of the calibration process of the volume and flow meters tested. Alternatively, they are used for comparing the performances of the apparatus with others when processing the same fluid and testing the same meters.

\section{Case study}

A new ultrasonic domestic meter is tested in the apparatus described in this work. The meter is able to measure air, natural gas and pure hydrogen. It has been developed by Pietro Fiorentini S.p.A. in the framework of Hy4Heat project [13]. Hy4Heat is a programme commissioned by the Department for Business, Energy \& Industrial Strategy of UK government. The aim is exploring the feasibility of replacing natural gas (methane) with hydrogen for domestic heating and cooking as a part of a plausible potential pathway for meeting heat decarbonization targets. In this context, it is necessary to triple the volume flow rate to transport the same quantity of energy as the higher heating value of hydrogen almost one third of that of natural gas. Therefore, the maximum volume flow rate for such domestic meter is set at $20 \mathrm{~m}^{3} / \mathrm{h}$ in case of hydrogen and $6 \mathrm{~m}^{3} / \mathrm{h}$ in case of natural gas. Such meter is able to measure volumes of gases or their volumetric flow rates.

After a calibration process, some units of this innovative meter were tested in the apparatus with hydrogen. All the samples were processed following the presented protocol. The result of the statistical process control for a sample of the session is shown in figure 5. It shows the Shewhart control chart, the moving average, the scatter plot, and the statistical indicators presented in the previous section. Then, the outcome of the data analysis consists of comparison between flow rate values, measured by the meter under test and the reference thermal mass flow meters. All the values are reported at standard conditions $\left(+15{ }^{\circ} \mathrm{C}\right.$ and $101.325 \mathrm{kPa}$ ). Finally, deviations are computed with equation 1 and illustrated in figure 6 . 

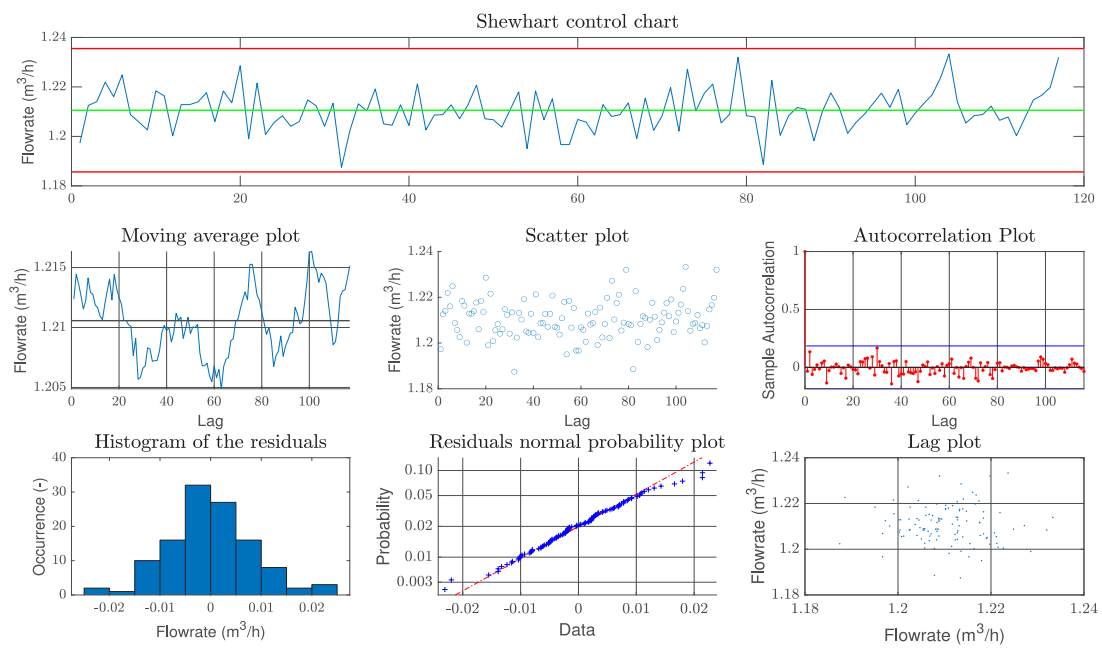

Fig. 5. Charts for statistical process control. They show: the Shewhart control chart, the moving average plot, the scatter plot and the presented statistical indicators.

The tested flow rates covered a range of $0.4-24 \mathrm{~m}^{3} / \mathrm{h}$ at three different temperatures: $+15{ }^{\circ} \mathrm{C}$ (the base temperature declared by the meter), $+55^{\circ} \mathrm{C}$ (the highest temperature covered by the new meter) and $-25^{\circ} \mathrm{C}$ (the lowest temperature covered by the new meter).

The indications for meters with gas temperature conversion device as Pietro Fiorentini's meters are from MID, OIML and EN14236 [6, 7, 9]. They impose the permissible error within $\pm 2.0 \%$ between the maximum flow rate and the transitional flow rate, and between $\pm 3.5 \%$ from the transitional flow rate and the minimum flow rate. In the case of hydrogen, the maximum flow rate is set to $20 \mathrm{~m}^{3} / \mathrm{h}$ and the transitional flow rate to $2 \mathrm{~m}^{3} / \mathrm{h}$. Moreover, the turndown ratio of the device must be at least 150 . Such performances must be retained in a range of $30{ }^{\circ} \mathrm{C}$, centred to the base temperature. The permissible errors are milder in case of higher or lower temperatures. In particular, for temperatures equal to $+55^{\circ} \mathrm{C}$ and $-25^{\circ} \mathrm{C}$ the error must be within $\pm 3.5 \%$ between the transitional flow rate and the maximum one and within $\pm 5.0 \%$ between the minimum flow rate and the transitional one.

As showed in figure 6 , the error trends at all temperatures are within the permissible error range for tests at $15^{\circ} \mathrm{C}$ (dashed dot lines). The error trends are also close to each other, showing no impact of temperatures in the outcome of the meters.

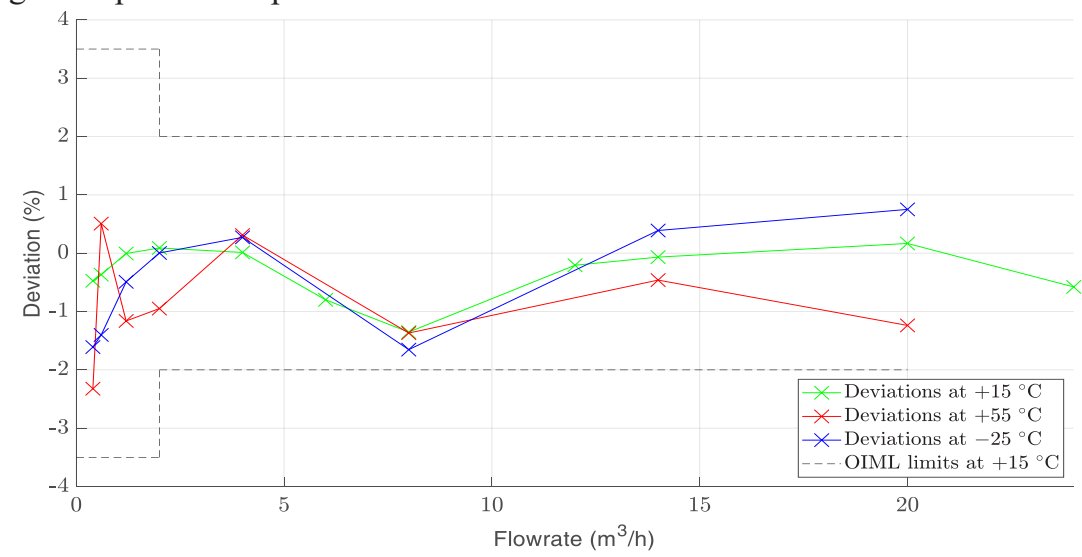

Fig. 6. Deviation trends of the meter under test with hydrogen at $+55^{\circ} \mathrm{C}$ (red line), $+15^{\circ} \mathrm{C}$ (green line), and $-25^{\circ} \mathrm{C}$ (blue line). The dashed lines show the permissible range for error trends in the $0-30{ }^{\circ} \mathrm{C}$ interval as declared by OIML $1371 \& 2$. Volumes are referred to standard unit $\left(15^{\circ} \mathrm{C}, 101.325 \mathrm{kPa}\right)$. 


\section{Conclusions}

The present work summarizes the development and construction of an experimental apparatus to test volume and flow meters in controlled environmental conditions. It also includes the implementation of a dedicated data analysis protocol. The apparatus has been validated for the calibration of volume and flow meters as presented in the case study. Consequently, the work comes to the following conclusions.

- The apparatus does not leak flammable gases and complies with the limit set by the indications in literature with a leakage equal to $0.000 \pm 1.1 \mathrm{~Pa} / \mathrm{min}$ [8].

- The apparatus is able to process all the flow rates needed as requested by the legislation for testing volume and flow meters [7].

- The climatic chamber can produce a stable environmental temperature in the volume and flow meters under test, also when processing high flow rates.

- The data analysis protocol allows the evaluation of the samples acquired and the production of results immediately after each test session, reducing the time required for the test campaign.

- The apparatus enabled the testing of hydrogen ultrasonic volume and flow meters at standard and extreme temperatures, they reported deviations lower than $3.5 \%$ between 0.4 and $2 \mathrm{~m}^{3} / \mathrm{h}$ and lower than $2 \%$ between 2 and $20 \mathrm{~m}^{3} / \mathrm{h}$, complying with the legislations at all temperatures [9]

Currently, the response of the apparatus is being compared with a different test bench in a certified testing facility. The comparison involves the same meters. They are tested in the same environmental conditions in a different testing facility. The future improvements may include a further thermal mass flow meter to improve the accuracy in the lowest flow range. Furthermore, they include an increase in the automation of the system introducing automated valves for flow regulation in section 3 of the apparatus. Ultimately, it is also expected a prosecution of the collaboration with Pietro Fiorentini S.p.A. for testing volume and flow meters. This will include tests with pure hydrogen and methane in the whole working temperature range of the meters under test.

\section{References}

1. B. Goldstein, D. Gounaridis, J.P. Newell, PNAS, 117, 19122-19130 (2020)

2. N.P. Brandon, Z. Kurban, R. Soc., 375 (2017)

3. H21, website https://h21.green/ (accessed on 06/07/2021)

4. International Energy Agency, The Netherlands 2020 (2020)

5. Presidenza del Consiglio dei Ministri, Piano Nazionale di Ripresa e Resilienza (2020)

6. European Parliament. Measuring Instruments Directive 2014/32/EU (2014)

7. EN14236:2018 (2018)

8. Physikalisch-Technische Bundesanstalt, Measuring Instruments for Gas, 29 (2003)

9. Organisation Internationale de Métrologie Légale, OIML R 137-1\&2 (2012)

10. D.C. Montgomery, Introduction to Statistical Quality Control (Wiley, 2013)

11. NIST, website https://www.itl.nist.gov/div898/handbook/ (accessed on 06/07/2021)

12. N.R. Tague, The Quality Toolbox (ASQ Quality press, Milwaukee, Wisconsin, 2005)

13. Hy4Heat, website https://www.hy4heat.info/ (accessed on 06/07/2021) 